\title{
Modeling and Simulation of Composite Materials
}

\author{
RAKESH K. BEHERA ®i, ${ }^{1,3}$ DINESH PINISETTY, ${ }^{2}$ \\ and DUNG D. LUONG ${ }^{1}$ \\ 1.-Composite Materials and Mechanics Laboratory, Mechanical and Aerospace Engineering \\ Department, Tandon School of Engineering, New York University, Brooklyn, NY 11201, USA. \\ 2.-Department of Engineering Technology, California State University Maritime Academy, 200 \\ Maritime Academy Dr., Vallejo, CA 94590, USA. 3.—e-mail: rakesh.behera@nyu.edu
}

Computational methods, ranging from electronic to continuum level, are extensively used in scientific and industrial sectors to investigate a wide range of material responses in a defined environment. While the computational community has a rich experience in examining metals, ceramics, and polymeric materials, the experience in simulating composites is still in its infancy. This difficulty in examining composites with computational approaches primarily stems from the following aspects, (1) composites are a combination of two or more dissimilar materials (e.g., metal/ceramic, metal/polymer, ceramic/polymer) which requires an accurate definition of not only the individual materials in the simulation approach but also the complex interfacial interactions, and (2) there is infinite possibility of designing unique composites by selecting different combinations of monolithic conventional materials, which demands accurate material parameters for providing meaningful computational insight for any given composite.

Based on the length-scale and time-scale in hand, continuum modeling, e.g., finite element modeling (FEM) is extensively used in simulating composite materials. In "Computational Modeling of the Mechanical Behavior of 3D Hybrid Organic-Inorganic Nanocomposites," Mohammad MaghsoudiGanjeh et al. have used FEM to model 3D hybrid nanocomposites to mimic biological ceramics such as bone and necre. The computational results conclude that the adhesive phase significantly enhanced the toughness of the organic-inorganic nanocomposite. In addition, the 3D architecture of the composite displays strain-hardening/softening behavior, which is absent from the $2 \mathrm{D}$ representation of the composite structure. The work by Tao

Rakesh Behera, Dinesh Pinisetty, and Dung Luong are guest editors for the Composite Materials Committee of the TMS Structural Materials Division, and organized the topic Modeling and Simulation of Composite Materials in this issue.
Huang et al. on "Establishment of Constitutive Relationships for Laminated Composites Considering the Variation of the Microhardness with the Strain of the Heterostructure Layers and Bonding Regions" further highlights the use of FEM to examine composites. In this work, FEM is used to verify the reliability of the theoretical model to determine the constitutive relationship of the heterostructure layers and bonding region of TA1/ Al1060/SS430 laminated composites.

Atomic-scale methods such as density functional theory (DFT) and molecular dynamics are generally used to gain a deeper fundamental understanding of the experimental system and can be used to generate better material parameters for larger lengthand time-scale modeling techniques. Yingang Gui et al. used DFT to analyze the $\mathrm{SOF}_{2}$ and $\mathrm{SO}_{2} \mathrm{~F}_{2}$ gas interactions on Ni-MoS ${ }_{2}$ monolayers. In the study "Effect of Nickel Doping on Adsorption of $\mathrm{SF}_{6}$ Decomposition Products Over $\mathrm{MoS}_{2}$ Surface," Yingang Gui et al. reported various electronic properties and established that the adsorption on $\mathrm{MoS}_{2}$ surface improves due to the presence of $\mathrm{Ni}$, which significantly increases the electrical conductivity. These results suggest $\mathrm{Ni}-\mathrm{MoS}_{2}$ can be used to stabilize $\mathrm{SF}_{6}$-insulated equipment.

In addition to the continuum- and atomic-scale computational approaches, theoretical methods have been proven to provide significant understanding of composite systems. Yasser Zare, and Kyong Yop Rhee predicted the tensile strength of polymer/carbon nanotube nanocomposites (PCNTs) using an expanded Takayanagi model. The theoretical model described in the "Evaluation of the Tensile Strength in Carbon Nanotubes-Reinforced Nanocomposites Using the Expanded Takayanagi Model" specifically assumes the strengthening and percolating efficiencies of the interphase between the polymer matrix and nanoparticles. The expanded Takayanagi model successfully calculated the average levels of the percolation threshold, 
interphase thickness, and interphase strength with respect to experiment for two representative PCNTs. Yasser Zare, and Kyong Yop Rhee also examined another nanocomposite in the article "A Simulation Work for the Influences of Aggregation/ Agglomeration of Clay Layers on the Tensile Properties of Nanocomposites". Combining a theoretical model with transmission electron microscopy, the authors discussed factors to reduce agglomeration of layered clay in polymer nanocomposites such as volume fraction, aspect ratio, and specific surface area of nanoparticles.

Again focusing on mechanical properties of composites, Liping Pan et al. designed a combination of numerical simulation with inverse algorithm to solve fracture-related properties for wedge splitting tests. In "Inverse Simulation of Fracture Parameters for Cement-Bonded Corundum Refractories", the authors have examined the influence of cement content and temperature on the fracture energy and tensile properties. The other theoretical article, entitled "Describing the Effective Conductivity of Two-Phase and Multiphase Materials via Weighted Means of Bounds and General Power Means", by Willi Pabst and Soňa Hř́balová focused on finding the effective thermal conductivity of multiphase materials. The recommendation of using appropriate means to describe materials with isotropic microstructure is presented in this article.

While computational and theoretical work are extensively used in examining composite materials, the work by Xianbo Xu and Nikhil Gupta presents a novel approach to obtain the mechanical properties of carbon fiber-reinforced laminated composites. For viscoelastic materials, dynamic mechanical analysis has been used to accelerate the testing process by transforming the measured viscoelastic properties to an elastic modulus. In their work "Artificial Neural Network Approach to Determine Elastic Modulus of Carbon Fiber-Reinforced Laminates,"Xianbo Xu and Nikhil Gupta made a significant improvement, where they leveraged an artificial neural network to build the master relationship of the storage modulus in three in-plane directions for addressing anisotropic structures with viscoelastic nature.

Finally, this topic includes an experimental investigation by Vasanth Chakravarthy Shunmugasamy and Bilal Mansoor. This work, entitled "Flexural Response of an Aluminum Foam Core/ Stainless Steel Facesheet Sandwich Composite," focuses on evaluating joint integrity using various experimental techniques (Vickers microhardness, nanoindentation, optical and scanning electron microscopy). From a three-point bend test experiment, the failure mechanism of the sandwich is reported to be core shear in nature, which matches well with the failure mode map established using Gibson's model. Therefore, this article uses both experimental and theoretical approaches to justify the failure mechanism obtained in the sandwichstructured composite.
The articles in this special topic on "Modeling and Simulation of Composite Materials" highlight the advantage of using computational methods to explore the complex nature of composite materials. To read or download any of the papers, follow the URL http://link.springer.com/journal/11837/71/11/p age/ 1 to the table of contents page for the November 2019 issue (vol. 71, no. 11).

- "Computational Modeling of the Mechanical Behavior of 3D Hybrid Organic-Inorganic Nanocomposites" by Mohammad MaghsoudiGanjeh, Liqiang Lin, Xiaodu Wang, Xianqiao Wang, and Xiaowei Zeng

- "Establishment of Constitutive Relationships for Laminated Composites Considering the Variation of the Microhardness with the Strain of the Heterostructure Layers and Bonding Regions" by Tao Huang, Mei Zhan, Yanbo Pei, Nan Xiang, Fangfang Yang, Yanyang Li, Junqing Guo, Xuewen Chen, and Fuxiao Chen

- "Effect of Nickel Doping on Adsorption of $\mathrm{SF}_{6}$ Decomposition Products over $\mathrm{MoS}_{2}$ Surface" by Yingang Gui, Hao Sun, Huangli Wei, Shukai Duan, Chao Tang, and Xiaoxing Zhang

- "Evaluation of the Tensile Strength in Carbon Nanotubes-Reinforced Nanocomposites Using the Expanded Takayanagi Model" by Yasser Zare and Kyong Yop Rhee

- "A Simulation Work for the Influences of Aggregation/Agglomeration of Clay Layers on the Tensile Properties of Nanocomposites" by Yasser Zare and Kyong Yop Rhee

- "Inverse Simulation of Fracture Parameters for Cement-Bonded Corundum Refractories" by $\mathrm{Li}$ Ping Pan, Zhu He, Yawei Li, Baokuan Li, and Shengli Jin

- "Describing the Effective Conductivity of TwoPhase and Multiphase Materials via Weighted Means of Bounds and General Power Means" by Willi Pabst and Soňa Hř́ibalová

- "Artificial Neural Network Approach to Determine Elastic Modulus of Carbon Fiber-Reinforced Laminates" by Xianbo Xu and Nikhil Gupta

- "Flexural Response of an Aluminum Foam Core/ Stainless Steel Facesheet Sandwich Composite" by Vasanth Chakravarthy Shunmugasamy and Bilal Mansoor

While individual length-scale and time-scale computational approaches provide extremely useful information, there is a need for an integrated multi-scale simulation approach to investigate existing and novel composite materials. Based on the current research efforts, we are hoping to see the development of multi-scale simulation approaches to examine composites in the near future.

Publisher's Note Springer Nature remains neutral with regard to jurisdictional claims in published maps and institutional affiliations. 